\title{
Fístula faringoesofágica tras paratiroidectomía por abordaje mínimamente invasivo*
}

\author{
Drs. JAIME RUIZ-TOVAR ${ }^{1}$, MIGUEL GRAS ${ }^{1}$, \\ GLORIA RODRÍGUEZ-VELASCO ${ }^{1}$,VIRGILIO FRESNEDA ${ }^{1}$ \\ 1 Servicio de Cirugía General y del Aparato Digestivo. \\ Hospital Universitario Ramón y Cajal. Madrid, España.
}

\begin{abstract}
Pharyngoesophageal fistula after minimally invasive parathyroidectomy

Introduction: Focused lateral approach is widely accepted for the surgery of solitary parathyroid adenomas, because it is a minimally invasive approach and its aesthetic advantages. Notwithstanding, when the pathlogical gland is not easily recognized, this approach difficults the search and makes more susceptible for iatrogenic lesions. Sometimes, PTH levels do not decrease up to $75 \%$ of initial values 15 minutes after the gland resection; a smaller decreasement do not exclude that the pathological gland has been already resected. Case report: A 69-years old man underwent a minimally invasive parathyroidectomy. Surgical act is complex, because of a difficult identification of the gland. In the postoperative course, the patient developed a pharyngoesophageal fistula that was successfully managed with a conservative approach.
\end{abstract}

Keywords: Parathyroidectomy, pharyngoesophageal fistula, focused lateral approach, PTH.

\section{Resumen}

Introducción: El abordaje unilateral en la cirugía del adenoma solitario de paratiroides está ampliamente aceptado por sus ventajas estéticas y ser un procedimiento mínimamente invasivo. Sin embargo, cuando la glándula patológica no es fácilmente detectada, dificulta mucho su búsqueda y facilita la iatrogenia. En ocasiones, los niveles de PTH no descienden al 75\% del valor inicial 15 minutos después de la paratiroidectomía; un descenso menor del $75 \%$ no excluye que la glándula patológica haya sido extirpada. Caso clínico: Varón de 69 años es sometido a paratiroidectomía a través de un abordaje mínimamente invasivo. La cirugía es compleja y prolongada al no identificarse fácilmente la glándula paratiroides. En el postoperatorio el paciente presenta una fístula faringoesofágica que se se maneja satisfactoriamente de forma conservadora.

Palabras clave: Paratiroidectomía, fístula faringoesofágica, abordaje unilateral, PTH.

\footnotetext{
Correspondencia: Dr. Jaime Ruiz-Tovar

Crta Colmenar Km 9,100. 28034 - Madrid, España.

E-mail: jruiztovar@gmail.com
}

* Recibido el 26 de Mayo de 2009 y aceptado para publicación el 11 de Septiembre de 2009. 


\section{Introducción}

La paratiroidectomía se recomienda actualmente en prácticamente todos los pacientes con hiperparatiroidismo primario, ya que la mayoría van a presentar síntomas y porque el tratamiento quirúrgico actual es más sencillo y rápido que en el pasado ${ }^{1-3}$. El abordaje unilateral consiste en el acceso directo sobre la glándula paratiroides patológica a través de una incisión de unos $2 \mathrm{~cm}$. Esta técnica ofrece ventajas estéticas, se puede realizar con anestesia local o mascarilla laríngea y precisa una mínima estancia hospitalaria, pero no está exenta de complicaciones $^{4,5}$. Presentamos un caso de fístula faringoesofágica tras paratiroidectomía por abordaje mínimamente invasivo.

\section{Caso clínico}

Varón de 69 años, con antecedentes de cólicos nefríticos de repetición, presenta calcemias elevadas $(11,1 \mathrm{mg} / \mathrm{dl})$ y niveles séricos de paratohormona (PTH) de 178,6 pg/ml. En una gammagrafía con ${ }^{99}$ Tecnecio-sestamibi se objetivó una glándula paratiroides inferior izquierda aumentada de tamaño e hipercaptante. Se decidió extirpar la glándula patológica. A través de una pequeña incisión de 3 $\mathrm{cm}$ en la cara lateral del cuello, se accedió a la celda tiroidea de forma lateral por fuera del músculo esternohioideo, quedando expuesta la zona teórica de la glándula paratiroides patológica. Se identificó una estructura que macroscópicamente parecía una glándula paratiroides aumentada de tamaño, se extirpó y se envió para estudio histológico intraoperatorio, siendo informado como tejido adiposo, aunque la muestra había llegado muy deteriorada. Los niveles de PTH séricos, 15 minutos después de la extirpación de esta estructura, fueron $100 \mathrm{pg} /$ $\mathrm{ml}$ (PTH preoperatoria $160 \mathrm{pg} / \mathrm{ml}$ ). Se continuó la búsqueda de la glándula paratiroides, enviando varias muestras intraoperatorias, no identificando tejido paratiroideo en ninguna de ellas. Durante la disección se visualizó mucosa esofágica, lo que indicaba una apertura accidental del esófago, suturándose la perforación con puntos sueltos. Aunque no se consiguió identificar un tejido correspondiente a la glándula paratiroides patológica, se desestimó la realización de una cervicotomía bilateral, por el elevado tiempo quirúrgico transcurrido $(150 \mathrm{~min})$ y el riesgo de producir mayor número de lesiones iatrogénicas, finalizando la intervención. El valor de la PTH postoperatoria fue de $90,1 \mathrm{pg} / \mathrm{ml}$.

En el postoperatorio el paciente comenzó con fiebre y salida de material purulento a través de la herida quirúrgica. Una radiografía de tórax mostró

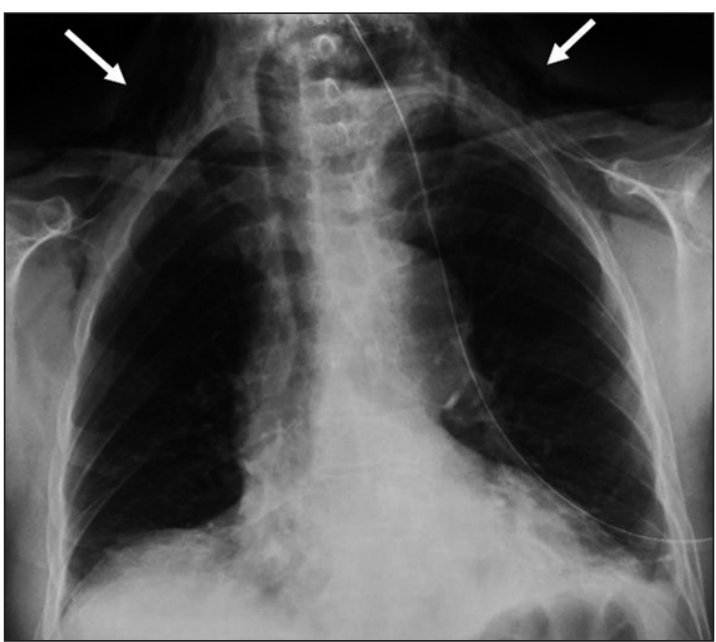

Figura 1. Radiografía de tórax. Enfisema subcutáneo.

un enfisema subcutáneo (Figura 1) y una TC cervical objetivó una colección de $3 \mathrm{~cm}$ en el lecho quirúrgico, con trayecto fistuloso hacia piel y contacto con el esófago, sugerente de fístula faringoesofágica (Figura 2). Se abrió la herida quirúrgica y se dejó un drenaje tipo Penrose, se suspendió la ingesta oral y se instauró nutrición parenteral durante 3 semanas. El paciente evolucionó satisfactoriamente; una cine-radiografía con contraste no mostró fístula faringoesofágica y el paciente comenzó la alimentación oral sin incidencias, siendo dado de alta a los 28 días de la intervención con niveles de calcio y PTH normales.

\section{Discusión}

La fístula faringoesofágica está ampliamente descrita como complicación postoperatoria de la cirugía de la columna cervical a través de un abordaje anterior, en el que es necesario disecar el espacio prevertebral, muy próximo a la cara esofágica posterior ${ }^{6}, \mathrm{y}$ en las laringuectomías, adyacentes a la cara anterior del esófago ${ }^{7}$. Sin embargo, la lesión faringoesofágica es un hecho inusual en la cirugía paratiroidea, ya que la cara lateral del esófago está teóricamente lejos del campo quirúrgico de la paratiroides. No obstante, en la intervención de nuestro paciente fue necesaria una mayor disección del área y una búsqueda en planos más profundos, lo que posibilitó la lesión esofágica.

Uno de los principales inconvenientes del abordaje lateral directo es el reducido campo operatorio; la incisión está dirigida para caer sobre la teórica localización de la glándula paratiroides, pero cuando ahí no se encuentra, la disección de tejidos vecinos 


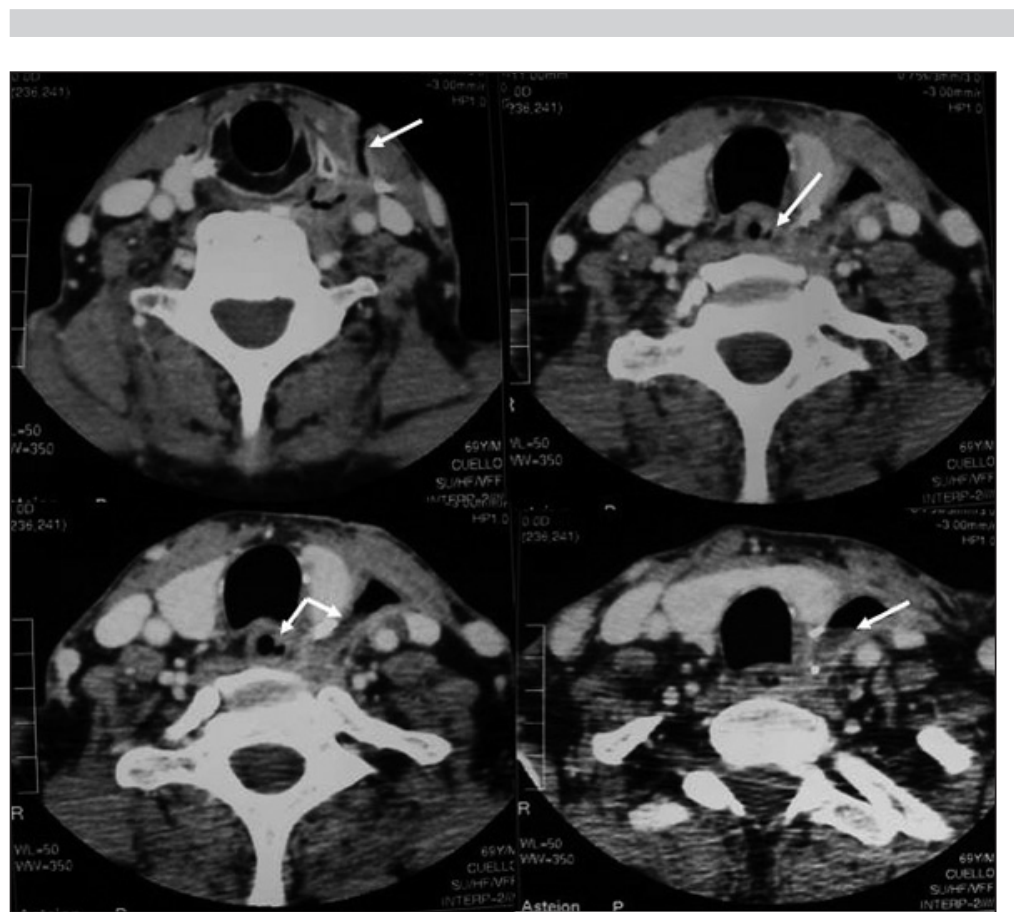

Figura 2. TC cervical. Colección en el lecho quirúrgico, con trayecto fistuloso hacia piel y contacto con el esófago.

resulta muy dificultosa, con escaso campo visual y ausencia de referencias anatómicas. Esos casos se consideran como exploraciones negativas y estaría indicado realizar una cervicotomía bilateral ${ }^{5}$. En nuestro paciente se desestimó la misma por el largo tiempo operatorio transcurrido y el riesgo de producir mayor iatrogenia. Así mismo, se observó un descenso de los niveles séricos de PTH, aunque no suficiente como para no intentar una búsqueda más exhaustiva en el mismo campo quirúrgico. Se acepta como suficiente un descenso de los niveles séricos de PTH del 75\% de los valores preoperatorios 15 minutos después de la extirpación de la paratiroides ${ }^{8}$. De todas maneras, la literatura estima en cerca de un 3\% de error en la correlación entre extirpación de adenoma de paratiroides y descenso del $75 \%$ de los niveles de PTH iniciales. Se ha observado que en ocasiones, aunque el descenso postoperatorio no alcance el $75 \%$, al repetir la determinación unas horas más tardes, éstas sí que alcanzan esos valores y el paciente se considera igualmente curado ${ }^{5,9}$. En nuestro paciente no se determinaron los niveles de PTH unas horas más tarde, pero al alta los niveles de PTH estaban dentro de la normalidad. La única explicación posible de esta normalización, es que la primera muestra enviada para estudio histológico efectivamente correspondiera con tejido paratiroideo, si bien como se encontraba en mal estado de conservación, no se pudo realizar un estudio correcto. Posiblemente este caso sea uno de esos en los que no se observa un descenso del $75 \%$ de los niveles de PTH a los 15 minutos de la extirpación de la glándula, pero sí posteriormente.

Las fístulas faringoesofágicas comienzan con dolor cervical, fiebre y signos inflamatorios en la cervicotomía. Habitualmente, la inflamación se limita a la región cervical y no progresa al mediastino, lo que condiciona que el estado general del enfermo no se deteriore. Sin embargo, ante la sospecha de infección, debe abrirse la herida y colocarse un drenaje, para evitar que la infección se propague al mediastino; debe instaurarse tratamiento antibiótico y nutrición parenteral. La mayoría de fístulas faringoesofágicas van a curar espontáneamente, como ocurrió en nuestro paciente, siendo necesario realizar un estudio radiológico con contraste que confirme el cierre de la fuga previo a la reintroducción de la alimentación por vía oral ${ }^{10}$.

\section{Referencias}

1. Sackett WR, Barraclough B, Reeve TS, Delbridge LW. Worldwide trends in the surgical treatment of primary hyperparathyroidism in the era of minimally invasive parathyroidectomy. Arch Surg 2002; 137: 1055-1059.

2. Sywak MS, Robinson BG, Clifton-Bligh P, Reeve TS, Barraclough BH, Fick GH, et al. Increase in presentations and procedure rates for hyperparathyroidism in Northern Sydney and New South Wales. Med J Aust 2002; 177: 246-249.

3. Utiger RD. Treatment of primary hyperparathyroidism. N Engl J Med 1999; 341: 1301-1302. 
4. Agarwal G, Barraclough B, Reeve TS, Delbridge LW. Minimally invasive parathyroidectomy using the "focused" lateral approach. Surgical technique. ANZ J Surg 2002; 72: 147-151.

5. Delbridge L. Minimally invasive parathyroidectomy: The Australian experience. Asian J Surg 2003; 26: 76-81.

6. ShenoY, Raja A. Delayed pharyngoesophageal perforation: Rare complication of anterior cervical spine surgery. Neurology India 2003; 5: 534-536.

7. Mäkitie AA, Niemensivu R, Hero M, Keski-Säntti H, Bäck L, Kajanti M, et al. Pharyngocutaneous fistula following total laryngectomy: a single institution's 10year experience. Eur Arch Otorhinolaryngol 2006; 263: 1127-1130.
8. Casanova Rituerto D. Táctica quirúrgica en el adenoma solitario. En: Sitges-Serra A, Sancho Insenser J. Guías Clínicas de la Asociación Española de Cirujanos. Cirugía Endocrina. Madrid: Aran, 1999: p.104.

9. Agarwal G, Barakate MS, Robinson B, Wilkinson M, Barraclough B, Reeve TS, et al. Intraoperative quick parathyroid hormone versus same day parathyroid hormone testing for minimally invasive parathyroidectomy: a cost-effectiveness study. Surgery 2001; 130: 963-970.

10. Parrilla Paricio P, Martínez de Haro LF, Ortiz Escandell A. Complicaciones de la resección esofágica. En: Parrilla Paricio P, Jaurrieta Mas E, Moreno Azcoitia M. Manual de la Asociación Española de Cirujanos. Madrid: Panamericana, 2005: 53-57. 